\title{
ARTICLE
}

Epidemiology

\section{Investigation of circulating metabolites associated with breast cancer risk by untargeted metabolomics: a case-control study nested within the French E3N cohort}

\author{
Elodie Jobard ${ }^{1,2}$, Laure Dossus ${ }^{3}$, Laura Baglietto ${ }^{4}$, Marco Fornilii ${ }^{4}$, Lucie Lécuyer ${ }^{5}$, Francesca Romana Mancini ${ }^{5}$, Marc J. Gunter ${ }^{3}$, \\ Olivier Trédan ${ }^{2}$, Marie-Christine Boutron-Ruault ${ }^{5}$, Bénédicte Elena-Herrmann ${ }^{1,6}$, Gianluca Severi ${ }^{5,7}$ and Joseph A. Rothwell $\mathbb{B D}^{5}$
}

\begin{abstract}
BACKGROUND: Perturbations in circulating metabolites prior to a breast cancer diagnosis are not well characterised. We aimed to gain more detailed knowledge to help understand and prevent the disease.

METHODS: Baseline plasma samples from 791 breast cancer cases and 791 matched controls from the E3N (EPIC-France) cohort were profiled by nuclear magnetic resonance (NMR)-based untargeted metabolomics. Partial least-squares discriminant analysis (PLS-DA) models were built from NMR profiles to predict disease outcome, and odds ratios and false discovery rate (FDR)-adjusted Cls were calculated for 43 identified metabolites by conditional logistic regression.

RESULTS: Breast cancer onset was predicted in the premenopausal subgroup with modest accuracy (AUC 0.61, 95\% CI: 0.49-0.73), and 10 metabolites associated with risk, particularly histidine (OR $=1.70$ per SD increase, FDR-adjusted $\mathrm{Cl} 1.19-2.41), \mathrm{N}$-acetyl glycoproteins ( $\mathrm{OR}=1.53$, FDR-adjusted $\mathrm{Cl} 1.18-1.97)$, glycerol (OR $=1.55$, FDR-adjusted $\mathrm{Cl} 1.11-2.18)$ and ethanol (OR $=1.44$, FDRadjusted $\mathrm{Cl}$ 1.05-1.97). No predictive capacity or significant metabolites were found overall or for postmenopausal women.

CONCLUSIONS: Perturbed metabolism compared to controls was observed in premenopausal but not postmenopausal cases. Histidine and NAC have known involvement in inflammatory pathways, and the robust association of ethanol with risk suggests the involvement of alcohol intake.
\end{abstract}

British Journal of Cancer (2021) 124:1734-1743; https://doi.org/10.1038/s41416-021-01304-1

\section{BACKGROUND}

Breast cancer is the most common cancer in women and accounts for around $25 \%$ of all female cancer cases worldwide. ${ }^{1}$ Alcohol intake, adult obesity and greater birthweight and height are reported to increase risk, while physical activity, breastfeeding and calcium intake have been linked with a decreased risk in population studies. ${ }^{2-4}$ Breast cancer is also a heterogeneous disease and risk factors vary between oestrogen receptor-positive and negative tumours ${ }^{5}$ and according to menopausal status. ${ }^{6}$

Knowledge of pre-diagnostic metabolism can potentially help identify population subgroups at greater risk and provide insight into the mechanisms of early carcinogenesis. A small number of studies have employed metabolomics previously to estimate associations prospectively between breast cancer risk and plasma or serum metabolite concentrations. ${ }^{7-13}$ Diverse study designs and analytical platforms have been employed, and although some broad common conclusions have been reached, such as the inverse associations between fatty acid-derived metabolites and breast cancer risk, important issues remain to be addressed.
Firstly, few of these studies have presented results by menopausal status, which may be an important determinant of both normal and pathological metabolic conditions. Secondly, alcohol intake is a likely risk factor for breast cancer, but most previous studies have relied on participant self-reported assessment. Although previous studies controlled for self-reported alcohol intake, few were able to include a biomarker surrogate for additional validation.

In this study, we test plasma metabolite associations with breast cancer risk using one of the largest metabolomics studies to date on the disease, in the French E3N cohort. Our study benefited from untargeted NMR metabolomics data that included the measurement of proxies of systemic inflammation ( $\mathrm{N}$-acetyl glycoproteins) and recent alcohol intake (free plasma ethanol), as well as a range of other endogenous metabolites. We aimed firstly to determine if full untargeted NMR plasma profiles were able to distinguish prediagnostic breast cancer cases from controls using multivariate predictive models, and secondly to test metabolite associations prospectively with breast cancer risk. Knowledge of how plasma metabolism is perturbed prior to a diagnosis may help disentangle

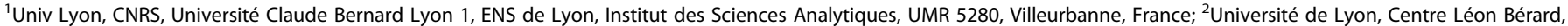

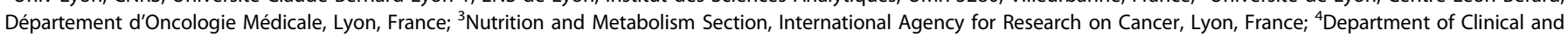

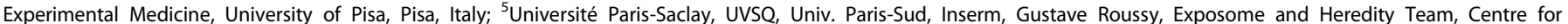

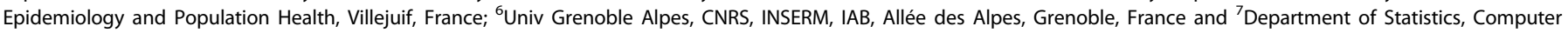
Science and Applications "G. Parenti" (DISIA), University of Florence, Firenze, Italy

Correspondence: Joseph A. Rothwell (joseph.rothwell@inserm.fr)

These authors contributed equally: Bénédicte Elena-Herrmann, Gianluca Severi, Joseph A. Rothwell

Received: 16 October 2020 Revised: 1 February 2021 Accepted: 4 February 2021

Published online: 15 March 2021 
the complex web of risk factors and translate into more effective disease prevention strategies.

\section{METHODS}

Study design and sample collection

The present study is based on a case-control study nested within the Etude Epidémiologique auprès de femmes de la MGEN (Mutuelle Générale de l'Education Nationale) cohort (E3N), a French multicentre prospective study designed to investigate risk factors for cancer and major non-communicable diseases in women. The cohort comprised nearly 100,000 women aged 40-65 years insured through a national health system and enroled from 1990 after returning baseline selfadministered questionnaires. ${ }^{14,15}$ Every 2-3 years after baseline, follow-up questionnaires were collected to update information about diet, lifestyle characteristics and medical events. These included detailed food-frequency questionnaires that allowed the calculation of alcohol intake in $\mathrm{g} /$ day. Blood samples were collected from around a quarter of all participants between 1994 and 1999. The E3N cohort was granted ethical approval by the French National Commission for Computed Data and Individual Freedom (Commission Nationale de I'Informatique et des Libertés) and all E3N participants provided written consent for the use of their blood samples and all data. E3N is the French component of the European Prospective Investigation into Cancer and Nutrition (EPIC), a collaborative study of over 500,000 participants in 10 countries coordinated by the International Agency for Research on Cancer, Lyon, France.

\section{Case ascertainment and matching}

Participants of E3N were asked to declare any new cancer event in periodic follow-up questionnaires. These reports were then investigated and validated by collecting pathological reports or clinical records from doctors. Tumour characteristics, such as stage, behaviour, histological subtype and hormone receptor status, were extracted from the reports. Incident breast cancer cases $(n=812)$ with available blood samples at baseline were identified. Each case was matched to a control who was free of cancer at the time of diagnosis. Matching factors were age at blood collection ( \pm 1 year), département (county) of residence (or collection centre), blood collection date ( \pm 9 months), menopausal status at blood collection date (pre- or post-menopausal) and fasting status (yes or no). After the exclusion of 7 breast cancer cases with no eligible control, 1610 plasma samples (805 cases and 805 matched controls) were retained.

Sample preparation and analysis

Blood samples were collected, processed and stored as previously described. ${ }^{16}$ To obtain plasma fractions, blood samples were recovered from citrate collection tubes and centrifuged at $1500 \times$ $g$ for $20 \mathrm{~min}$. The samples were then stored in liquid nitrogen at $-196^{\circ} \mathrm{C}$ until laboratory analysis. To check data quality and reproducibility, two quality control plasma samples (QC) collected from one healthy donor were prepared in parallel with experimental samples. These were placed at the beginning and end of each sample rack to evaluate analytical variability.

One-dimensional ${ }^{1} \mathrm{H}-\mathrm{NMR}$ spectra were acquired on a Bruker Avance III spectrometer (Bruker, Rheinstetten, Germany) operating at $600.55 \mathrm{MHz}$ and equipped with a temperature-controlled automatic sample changer and $5-\mathrm{mm}$ TCl cryo probe. Standard ${ }^{1} \mathrm{H}$-NMR pulse sequences, NOESY and CPMG with water presaturation, were applied to samples to generate raw spectra (Supplementary Fig. S1). The NOESY mixing time was set to $100 \mathrm{~ms}$ and the total CPMG filter to $80 \mathrm{~ms}$ for efficient attenuation of the lipid NMR signals. These spectra were manually phased and baseline-corrected before being imported into Bruker Amix software for processing. Spectra were reduced, over a chemical shift range of $0.5-9.0 \mathrm{ppm}$, to 8500 bins, each of which was integrated as a separate variable.
Two-dimensional NMR spectra were additionally acquired from one case and one control sample to assign NMR signals observed in the ${ }^{1} \mathrm{H}$ one-dimensional profiles to metabolite identities. Fiftysix identities were assigned from interactive analysis of this dataset and reference to NMR shift knowledge bases (Supplementary Table S1). For metabolite quantification, NMR chemical shift regions were grouped into 243 buckets that corresponded to reconstructions of peak entities. Clusters of variables corresponding to the same metabolite were then summed to give a single intensity, resulting in 43 measurements that corresponded to distinct metabolites or metabolite classes. Full details are given in Supplementary Information.

\section{Statistical analysis}

After the exclusion of 28 participants for which spectra did not meet quality control checks, 1582 remained for statistical analysis. Relative standard deviations (RSD) were calculated for each chemical shift region as a check of analytical reproducibility, and the PC-PR2 method was employed to assess the effect of different variables upon metabolomics data. ${ }^{17}$ Predictive models for sample classification were fit using partial least-squares discriminant analysis (PLS-DA) based on full untargeted NMR profiles. Models were fit for all participants, by menopausal status at blood collection ( $n=354$ and 1218 for pre- and post-menopausal, respectively), age category at diagnosis (over or under 55 years), fasting status at blood collection (yes or no) and time to diagnosis (within 2 or 5 years of blood collection).

NMR variables were transformed to the residuals of a linear model of metabolite intensity on blood collection centre, week of laboratory analysis, biobank storage time, waiting time to sample fractionation and fasting status. ${ }^{18}$ This matrix of residuals was used to fit a partial least-squares discriminant analysis (PLS-DA) model with case-control status as a binary response. Models were trained and refined on a random $75 \%$ of these data and tested on the remainder, and this final model used to predict case-control status for the test observations. Accuracy, as receiver-operating characteristic (ROC) area under the curve (AUC), was used to assess model performance. Analyses were performed using $\mathrm{R}$ statistical software, version 3.5.2 and PLS-DA models were fit using the caret package. ${ }^{19}$

Odds ratios (OR) and $95 \%$ confidence intervals were then calculated for each of the 43 annotated metabolites using conditional logistic regression. The highest and lowest $1 \%$ of intensities were first excluded for each metabolite and data were modelled as continuous variables with odds ratios corresponding to a one-SD increase in relative concentration. Models were adjusted for sample waiting time before fractionation, BMI, diabetes status, sample-storage time, waist-to-hip ratio, daily alcohol intake ( $\mathrm{g} /$ day) and duration of use of menopause hormonal treatment at blood collection. To account for multiple testing, $P$-values were adjusted using the false discovery rate (FDR) procedure and the significance threshold set at 0.05 . For those associations that were significant by these criteria, FDR-adjusted confidence intervals $(\mathrm{Cl})$ for ORs were also calculated using the method of Benjamini and Yekutieli. ${ }^{20}$

The relationship between reported alcohol intake and plasma ethanol was investigated on a continuous basis by Pearson correlation and for quartiles of alcohol intake by the Kruskal-Wallis one-way analysis of variance. As a sensitivity analysis and further investigation of alcohol intake in relation to breast cancer, the premenopausal metabolite risk models were repeated treating plasma ethanol concentration as a measure of exposure to alcohol, adjusting for this variable in all other metabolite models.

A sensitivity analysis was carried out excluding non-fasting matched pairs overall and by menopausal status. For the premenopausal subgroup only, further sensitivity analyses were performed adjusting for lifetime alcohol intake pattern and excluding cases diagnosed in the first two years after blood collection and their corresponding controls. 
Table 1. Characteristics of the breast cancer metabolomics case-control study participants.

\begin{tabular}{|c|c|c|c|}
\hline \multirow[t]{2}{*}{ Baseline characteristic } & \multicolumn{2}{|c|}{ Mean \pm SD or frequency (\%) } & \multirow[b]{2}{*}{$P$-value ${ }^{\mathrm{a}}$} \\
\hline & $\begin{array}{l}\text { Controls } \\
(N=791)\end{array}$ & $\begin{array}{l}\text { Cases } \\
(N=791)\end{array}$ & \\
\hline Age at blood collection (years) & $56.8 \pm 6.6$ & $56.8 \pm 6.6$ & - \\
\hline \multicolumn{4}{|l|}{ Menopausal status at blood collection } \\
\hline Pre-menopausal & $180(22.5)$ & $179(22.5)$ & \multirow[t]{2}{*}{-} \\
\hline Post-menopausal & $611(77.5)$ & $612(77.5)$ & \\
\hline \multicolumn{4}{|l|}{ Fasting status at blood collection } \\
\hline Fasting & $287(36.3)$ & $287(36.3)$ & \multirow[t]{2}{*}{-} \\
\hline Non-fasting & $504(63.7)$ & $504(63.7)$ & \\
\hline \multicolumn{4}{|l|}{ Follow-up time to cancer diagnosis } \\
\hline 5 years or less & - & $412(52.1)$ & \multirow[t]{2}{*}{-} \\
\hline More than 5 years & - & $379(47.9)$ & \\
\hline \multicolumn{4}{|l|}{ BMI $\left(\mathrm{kg} / \mathrm{m}^{2}\right)$} \\
\hline Underweight or normal $(<25)$ & $538(68.3)$ & $564(71.4)$ & \multirow[t]{4}{*}{0.27} \\
\hline Overweight $^{25-30}$ & $204(25.9)$ & $177(22.4)$ & \\
\hline Obese $(\geq 30)$ & $46(5.8)$ & $49(6.2)$ & \\
\hline Unknown & $3(0.4)$ & $1(0.1)$ & \\
\hline \multicolumn{4}{|l|}{ Waist to hip ratio } \\
\hline$<0.8$ & $589(74.8)$ & $573(72.6)$ & \multirow[t]{3}{*}{0.33} \\
\hline$\geq 0.8$ & $198(25.2)$ & $216(27.4)$ & \\
\hline Unknown & $4(0.5)$ & $2(0.3)$ & \\
\hline \multicolumn{4}{|l|}{ Smoking status } \\
\hline Yes & $66(8.3)$ & $62(7.8)$ & \multirow[t]{2}{*}{0.78} \\
\hline No & $725(91.7)$ & $724(92.2)$ & \\
\hline \multicolumn{4}{|l|}{ Diabetic status } \\
\hline Yes & $32(4.0)$ & $27(3.4)$ & \multirow[t]{2}{*}{0.6} \\
\hline No & $759(96.0)$ & $764(96.6)$ & \\
\hline Reported alcohol intake, g/day & $11.7 \pm 15.0$ & $12.6 \pm 15.4$ & 0.25 \\
\hline \multicolumn{4}{|l|}{ Lifetime alcohol drinking pattern } \\
\hline Non-consumers ( $0 \mathrm{~g} /$ day) & $153(19.5)$ & $146(18.5)$ & \multirow[t]{4}{*}{0.48} \\
\hline Light consumers (1-10 g/day) & $334(42.5)$ & $321(40.6)$ & \\
\hline Drinkers (>10 g/day) & $298(38.0)$ & $323(40.9)$ & \\
\hline Unknown & $6(0.8)$ & $1(0.1)$ & \\
\hline \multicolumn{4}{|l|}{ Blood pressure status } \\
\hline Normal & $642(81.8)$ & $653(83.0)$ & \multirow[t]{3}{*}{0.55} \\
\hline Hypertensive & $143(18.2)$ & $133(17.0)$ & \\
\hline Unknown & $6(0.8)$ & $5(0.6)$ & \\
\hline \multicolumn{4}{|l|}{ Previous use of oral contraceptives } \\
\hline Yes & $768(97.7)$ & $769(97.8)$ & \multirow[t]{2}{*}{1} \\
\hline No & $18(2.3)$ & $17(2.1)$ & \\
\hline Previous breastfeeding & & & \\
\hline Yes & $493(63.9)$ & $472(61.1)$ & 0.26 \\
\hline No & $278^{36}$ & $301(39.0)$ & \\
\hline Unknown & $6(0.8)$ & $5(0.6)$ & \\
\hline $\begin{array}{l}\text { Menopause hormone therapy use at bloo } \\
\text { collection (post-menopausal) }\end{array}$ & & & \\
\hline Yes & $371(46.9)$ & $416(52.6)$ & 0.01 \\
\hline No & $240(30.3)$ & $304(24.8)$ & \\
\hline $\begin{array}{l}\text { Duration of use of menopause hormonal } \\
\text { treatment, years (post-menopausal) }\end{array}$ & $3.9 \pm 4.5$ & $4.4 \pm 4.7$ & 0.06 \\
\hline
\end{tabular}

\section{RESULTS}

Participant and tumour characteristics

Baseline characteristics of the metabolomics study participants are shown in Table 1 and Supplementary Table S2A. Most cases (77\%) were post-menopausal at blood collection and the median time to diagnosis was 4.75 years (range 0.01-12.67 years) (Fig. 1a). Cases were more frequently users of menopause hormone therapy at the time of blood collection. Most tumours were invasive ductal carcinomas and were oestrogen receptor (ER) positive (Supplementary Table S2B). Cases who were pre- and post-menopausal at blood collection did not differ in tumour subtype, behaviour, grade or ER status, and differed slightly only in the proportion of progesterone receptor- positive cases. Only 8 cases that were premenopausal at blood collection were reported to be postmenopausal at breast cancer diagnosis.

Association of untargeted profiles and individual metabolites with breast cancer risk

The processing of raw NMR spectra produced a matrix of 8500 chemical shift regions, with a median RSD of $6.9 \%$ across QCs (Supplementary Fig. S1). A median RSD in intensity of $6.9 \%$ among chemical shift regions (interquartile range: 1.3-18.7\%) indicated that the analysis was reproducible and the data of high precision (Supplementary Fig. S2). BMl, diabetes status and collection centre accounted for most variability in raw metabolomics data (Supplementary Fig. S3). The PLS-DA model discriminated breast cancer cases from controls at blood collection modestly in premenopausal participants (ROC AUC $=0.61,95 \% \mathrm{Cl}$ 0.49-0.73). For all other subgroups tested, an AUC of 0.5 was comfortably within $95 \% \mathrm{Cls}$, indicating predictions no better than random chance (Table 2).

The 43 metabolites or biological indicators annotated according to 2D NMR chemical shift patterns comprised small alcohols and ketones, amino acids and other $\mathrm{N}$-containing metabolites, organic acids, plasma proteins, cholines and three groups of fatty acids with distinct spectral characteristics. Ethanol was the only metabolite of direct exogenous origin, although no correlation was observed between plasma ethanol and reported alcohol intake on a continuous $(r=-0.03)$ or categorical basis $(P=0.70$ for quartiles of reported alcohol intake, Fig. 1b). Metabolites clustered strongly by correlation and concentrations of fatty acids were inversely correlated with those of other metabolites overall (Fig. 1c). Specific groups of metabolites, such as the branchedchain amino acids valine and leucine, were highly intercorrelated.

In the whole study, concentrations of N-acetyl glycoproteins (NAC), ethanol, hypoxanthine and dimethylamine, were positively associated with risk of breast cancer, although these associations were not significant after controlling for the false discovery rate ( $P=0.162$ and 0.351 , respectively) (Fig. 2). In the premenopausal group however, breast cancer risk was associated with an increase in the concentrations of 10 metabolites after FDR adjustment for multiple testing. The strongest associations were observed for histidine $(O R=1.70$ per SD increase in concentration, FDR-adjusted $\mathrm{Cl}$ 1.19-2.41), glycerol $(\mathrm{OR}=1.55$ per SD increase, FDR-adjusted $\mathrm{Cl} 1.11-2.18)$, NAC $(O R=1.53$ per SD increase, FDR-adjusted $\mathrm{Cl} 1.11-2.11)$ and ethanol ( $O R=1.44$ per SD increase, FDR-adjusted $\mathrm{Cl} 1.05-1.97)$. Two of the fatty acid groups (mainly LDL and mainly VLDL) were borderline inversely associated with breast cancer risk (FDRadjusted $P=0.062$ ). Table 3 shows the results for those metabolites with raw $P$-values $<0.05$ in at least one of the study groups. ORs and $P$-values for all metabolites are given overall and for pre- and postmenopausal subgroups in Supplementary Tables S3-S5, respectively.

In the premenopausal subgroup, the additional adjustment for plasma ethanol concentration caused most metabolite associations to be weakened (Table 3). Only histidine remained significant after multiple testing adjustment $(\mathrm{OR}=1.58$ per $\mathrm{SD}$ increase in concentration, FDR-adjusted $\mathrm{Cl} 1.18-2.12$ ). Acetone was the only metabolite to increase in strength of association but did not meet the $P$-value significance threshold. In other sensitivity analyses in this group, additional adjustment for lifetime alcohol drinking did not appreciably affect associations, and associations weakened or were no longer statistically significant when those cases who were diagnosed within 2 years of blood collection were excluded (Supplementary Table S6). No statistically significant associations were found in the ER+ subgroup. 
a

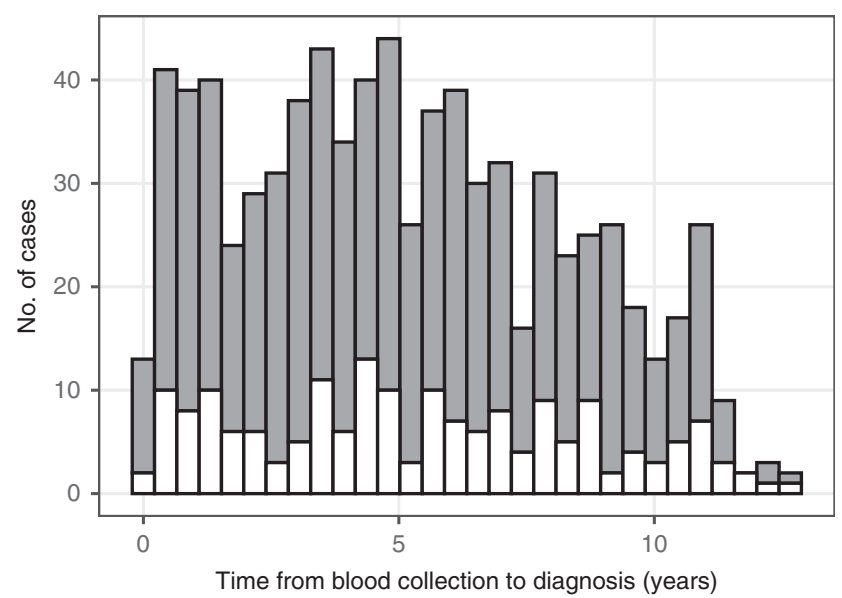

b

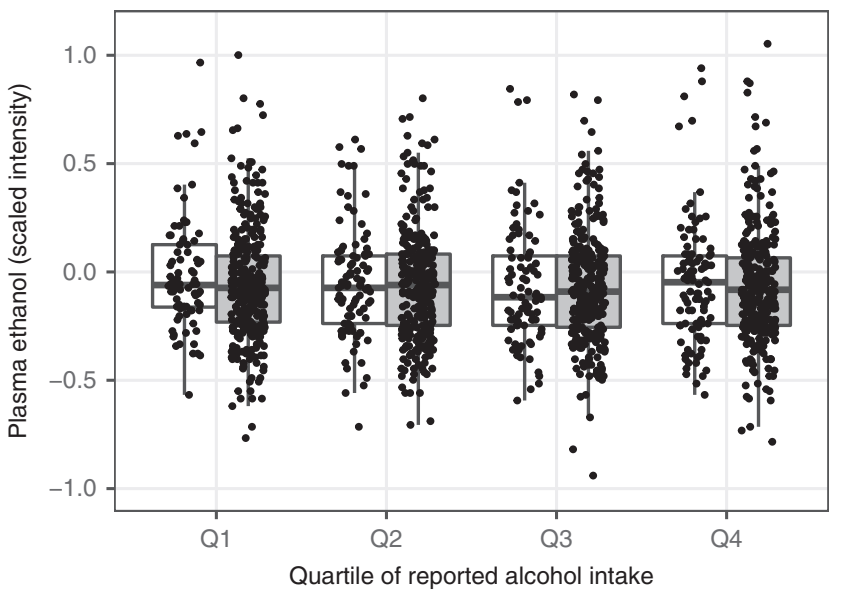

C

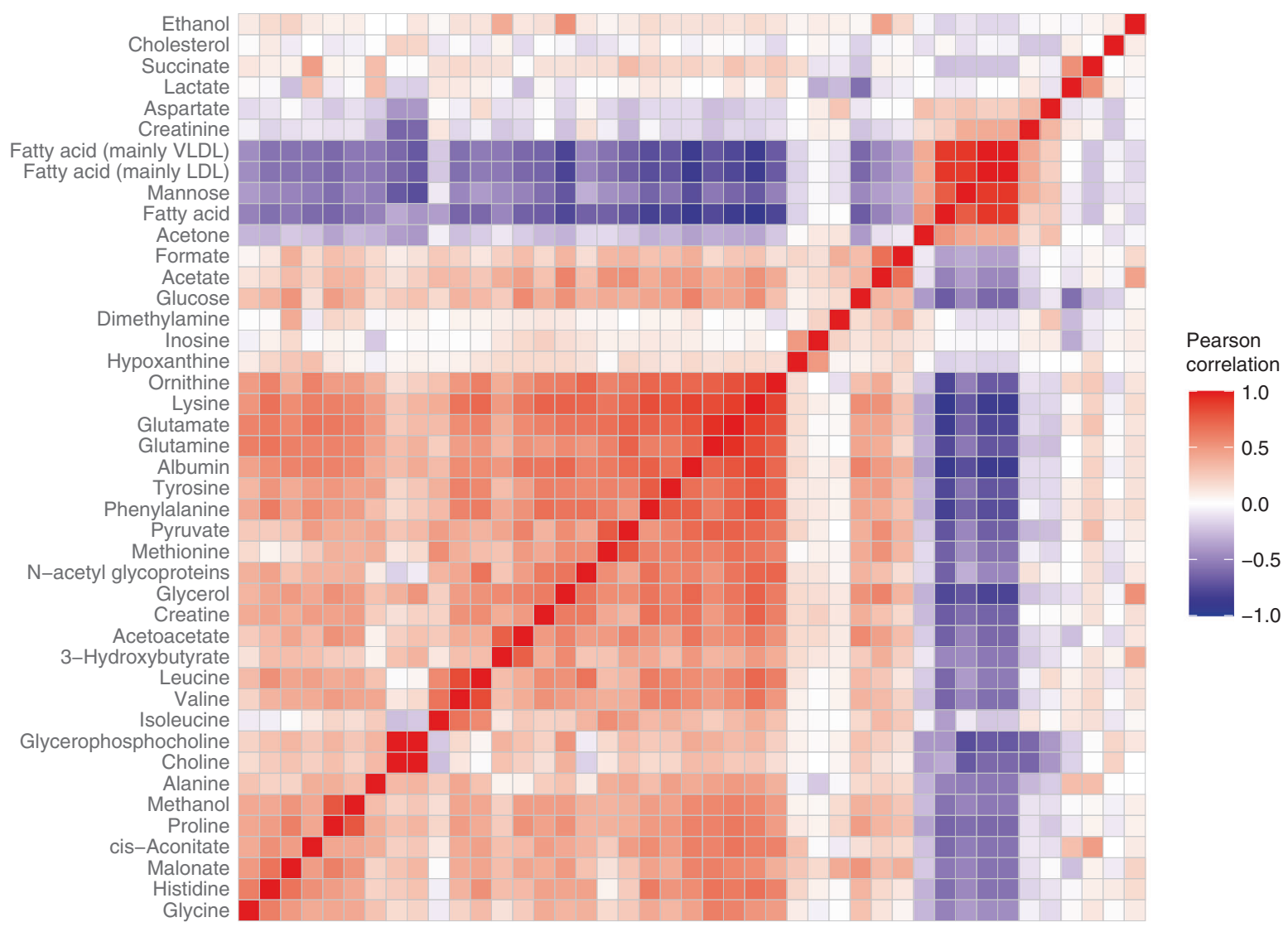

Fig. 1 Descriptive analysis of participant and metabolite data. a Distribution of follow-up times to diagnosis. b Relationship between reported alcohol intake and plasma ethanol concentrations. c Plasma metabolites identified and their correlations. No significant association was found between quartile of alcohol intake and plasma ethanol concentration (Kruskal-Wallis $P>0.05$ for pre- and postmenopausal groups). Correlations were calculated from fasted participants only and metabolites are ordered by hierarchical cluster as determined by Ward's method.

The exclusion of non-fasting participants (around two-thirds overall) attenuated ORs in some cases, particularly in the postmenopausal subgroup. Most notably, total fatty acids became suggestively associated with breast cancer risk (OR $=1.32$ per SD increase, $95 \% \mathrm{Cl} 1.03-1.67$, FDR-adjusted $P=0.14$ ). In the premenopausal subgroup, ORs for fasting participants remained unchanged or increased for histidine, NAC and ethanol, valine and leucine, although the association of glycerol with risk was diminished. $P$-values no longer met the FDR threshold due to the limited number of participants in these subgroups. ORs and $P$-values for all metabolites are given overall and for pre- and postmenopausal subgroups by fasting status in Supplementary Tables S7-S9, respectively. A comparison of overall and fasting data for pre- and postmenopausal subgroups is presented in Supplementary Fig. S4. 
Table 2. Receiver operating curve AUCs for prediction of breast cancer from untargeted NMR features.

\begin{tabular}{|c|c|c|c|c|}
\hline \multicolumn{5}{|l|}{ Menopausal status at blood collection } \\
\hline Pre-menopausal & 354 & 5 & 5-fold, 5 times & $0.61(0.49-0.73)$ \\
\hline Post-menopausal & 1218 & 4.77 & 10-fold & $0.53(0.46-0.59)$ \\
\hline$<55$ years & 265 & 2.7 & 5-fold, 5 times & $0.63(0.49-0.77)$ \\
\hline$\geq 55$ years & 1307 & 5.48 & 10-fold & $0.49(0.43-0.55)$ \\
\hline \multicolumn{5}{|l|}{ Fasting status at blood collection } \\
\hline Yes & 572 & 4.73 & 10-fold & $0.52(0.43-0.62)$ \\
\hline No & 1000 & 4.96 & 10-fold & $0.53(0.46-0.61)$ \\
\hline \multicolumn{5}{|l|}{ Time from blood collection to cancer diagnosis } \\
\hline
\end{tabular}

\section{DISCUSSION}

In this study, full NMR profiles of baseline plasma samples were able to discriminate between breast cancer cases and controls in premenopausal women only. Although no individual metabolite was significantly associated with breast cancer risk overall after FDR adjustment of $P$-values, 10 metabolites were positively associated with risk in the premenopausal subgroup, particularly histidine, glycerol, NAC and ethanol. Since no clear associations were found between metabolites and breast cancer risk in the larger postmenopausal subgroup, our study is the first to report differential metabolite associations with breast cancer by menopausal status.

Endogenous oestrogen production decreases substantially following the menopause and, since breast cancer is considered a hormone-dependent neoplasm, ${ }^{6,21}$ risk factors may vary according to menopausal status. However, previous studies on breast cancer and metabolomics have used predominantly or wholly postmenopausal participants. These studies usually reported inverse associations between disease risk and blood triglycerides, fatty acids or their derivatives, ${ }^{8-11}$ suggesting that the disease is preceded by a marked dyslipidaemia. Amino acids, conversely, were most commonly found to be positively associated with breast cancer risk, among them the branchedchain amino acids valine and leucine, as well as lysine, arginine, phenylalanine and glutamine. ${ }^{12,13}$ Multiple studies also found carnitine derivatives to be positively associated with the disease. ${ }^{9,12,22}$ Findings reported by menopausal status are scarce. In those studies that included premenopausal participants, case numbers were low and heterogeneity by menopausal status was either not detected for metabolites that were associated with breast cancer overall ${ }^{9}$ or not specifically examined. ${ }^{11,13}$

In our study, no metabolites met the $P$-value threshold for significance in the postmenopausal subgroup although some associations strengthened upon the exclusion of non-fasting participants and fatty acids in particular approached significance for a positive association with breast cancer risk. This finding appears contrary to those of previous studies, although our NMRbased method did not resolve individual fatty acids, which may elicit opposing bioactivities. ${ }^{23}$ In the premenopausal subgroup, several statistically significant associations were detected, the strongest of which was the $\mathrm{N}$-acetylation of glycoproteins (NAC). NAC is involved in the activation of inflammatory pathways and is a robust indicator of systemic inflammation similar to C-reactive protein. ${ }^{24}$ Although not measured previously in prospective studies on breast cancer, it has been associated with an increased risk of all-cause and cancer-specific mortality. ${ }^{25}$ Also positively associated with risk was histidine, which has previously been implicated in breast and colorectal cancer as a necessary precursor of histamine, whose release is an early event in inflammatory responses and that is a regulator of cell proliferation. ${ }^{26}$ Other notable positive associations were observed for glutamate, which has previously been linked to insulin resistance ${ }^{27}$ and glycerol, which is perturbed in conditions of dyslipidaemia. ${ }^{28}$ The reduction is oestrogen levels post menopause and the associated physiological changes likely drive the differences in metabolite associations by menopausal status. A recent study suggested that menopause attenuates metabolism, particularly lipids and inflammatory biomarkers, independently of advancing age. ${ }^{29}$ Thus, our results on a relatively small proportion of the metabolome might reflect complex interactions between these factors and metabolic changes related to early carcinogenesis.

Alcohol intake is considered a risk factor for breast cancer in both pre- and postmenopausal women. ${ }^{30}$ In the full E3N cohort, associations were found for postmenopausal breast cancer risk only ${ }^{31}$ and the metabolomics subset under study was representative in this respect with a borderline positive association per $10 \mathrm{~g}$ of alcohol intake in postmenopausal women and no association in premenopausal women (data not shown). Two previous studies have linked circulating ethanol to breast cancer risk, predominantly in postmenopausal women: an NMR metabolomics study that found ethanol to be part of a profile predictive of disease development ${ }^{7}$ and a nested case-control study within the PLCO cohort that found ethanol glucuronide, a known biomarker of alcohol intake, to be associated with overall risk, as well as other metabolites originating from alcoholic drinks. ${ }^{10}$ Multiple mechanisms have been proposed to link ethanol and breast cancer risk. A portion of absorbed ethanol is converted by alcohol dehydrogenase to acetaldehyde, a carcinogen that promotes tumorigenesis by forming DNA adducts. ${ }^{32}$ Exposure to 
a

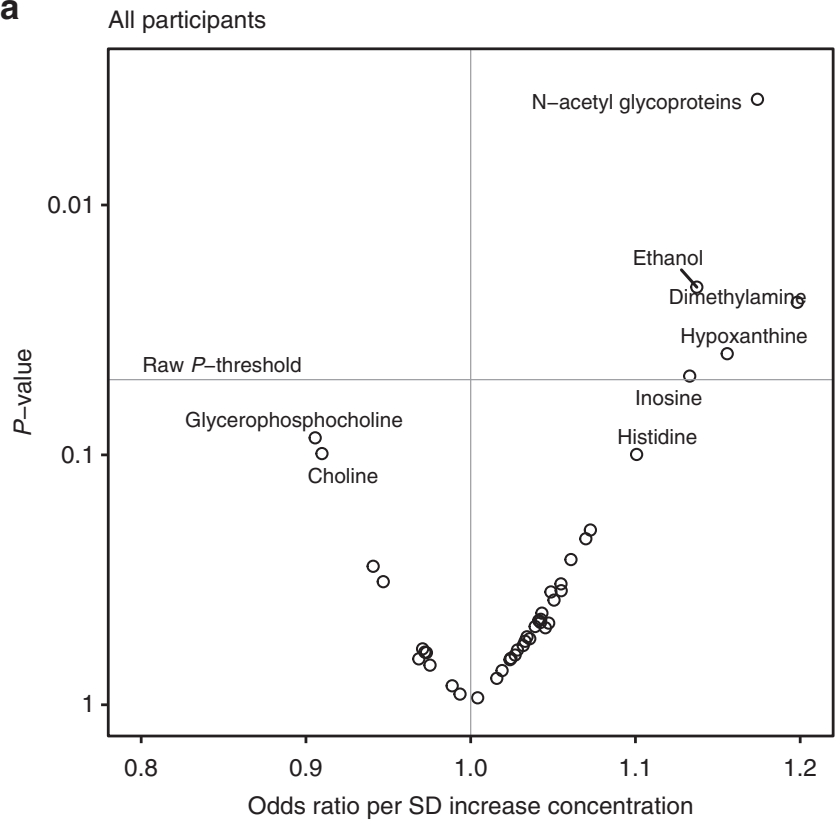

C

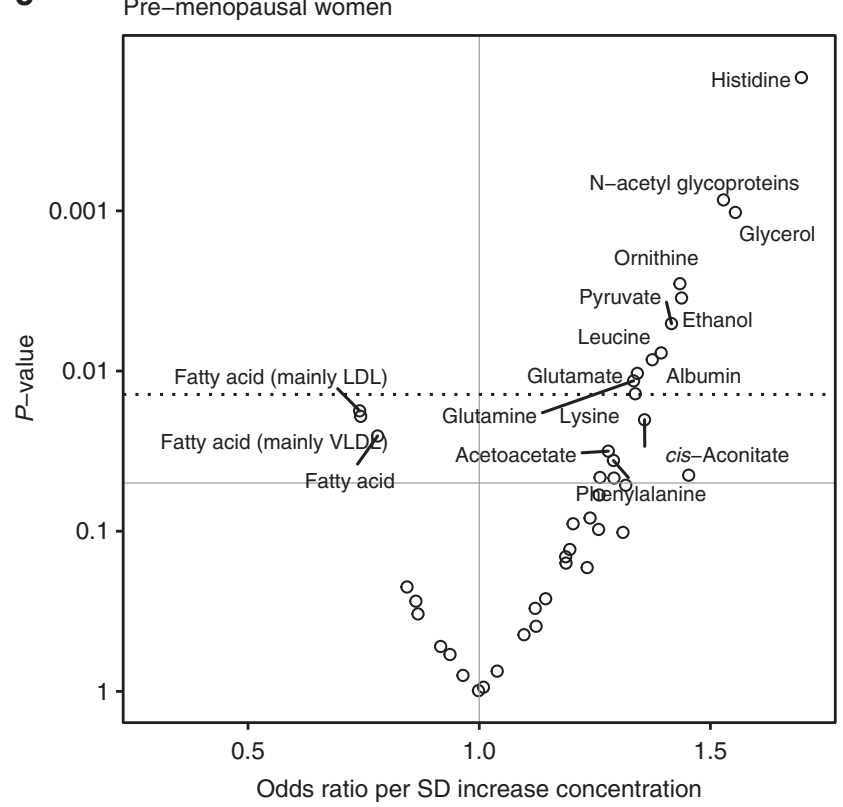

b

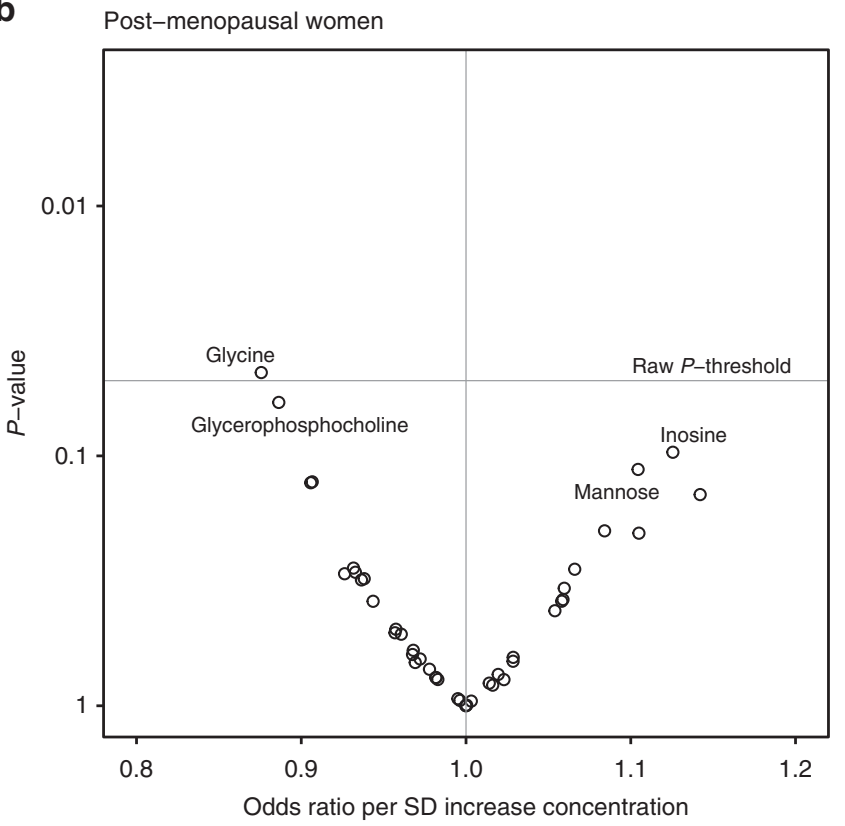

d

Pre-menopausal, adjusted for plasma ethanol

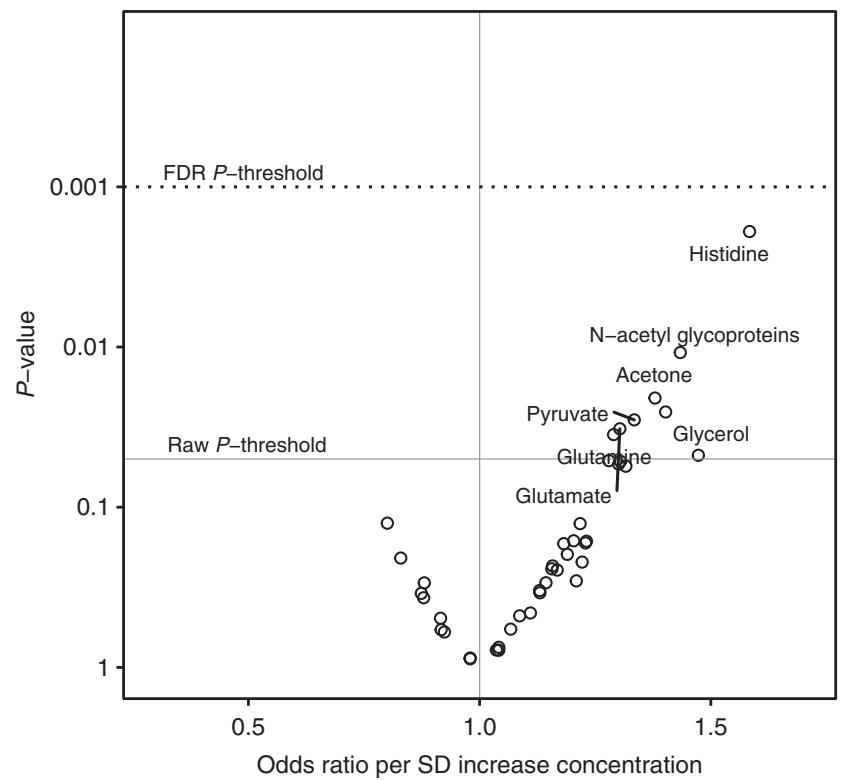

Fig. 2 Odds ratios versus $\boldsymbol{P}$-values ('smile plots') for metabolite univariate risk models by subgroup. a All study participants. b Postmenopausal women only. c Pre-menopausal women only. d Pre-menopausal women only and adjusted for plasma ethanol concentration. Raw $P$-values corresponding to FDR $P$-value thresholds could not be determined for $(\mathbf{a}, \mathbf{b})$. Models were adjusted for sample waiting time before plasma fractionation, BMI, diabetes status, waist-to-hip ratio, storage time, blood pressure, daily alcohol intake and duration of use of menopause hormonal treatments at blood collection. Five case-control pairs not matched on menopausal status were excluded from the analysis.

ethanol is known to disrupt endogenous metabolism. For example, blood lipids such as glycerophospholipids are perturbed by high alcohol intake, ${ }^{33-35}$ and amino acid profiles were perturbed by heavy alcohol intake in Japanese men. ${ }^{36}$ Levels of branched-chain amino acids, including leucine, were seen to increase in response to high wine intake. ${ }^{37}$ Metabolic changes due to alcohol intake are broad since regular ethanol exposure may disrupt the growth of gut microbiota and thus affect nutrient absorption, cause hepatocyte damage $^{38}$ and react directly with endogenous metabolites.

We observed a strong association between plasma ethanol and premenopausal breast cancer risk only, in contrast to the associations for self-reported alcohol intake, and no detectable correlation between plasma ethanol and alcohol intake. Assuming our measurements of free plasma ethanol were representative of overall blood ethanol, this suggests that premenopausal cases consumed more alcohol than controls in the hours preceding blood collection even though no difference was observed in reported intakes between cases and controls. Also, the selfreporting of alcohol intake has been suggested to be subject to bias in observational studies. ${ }^{39}$ In addition, ethanol was strongly positively correlated with some amino acids and inversely correlated with overall fatty acids, and associations between 


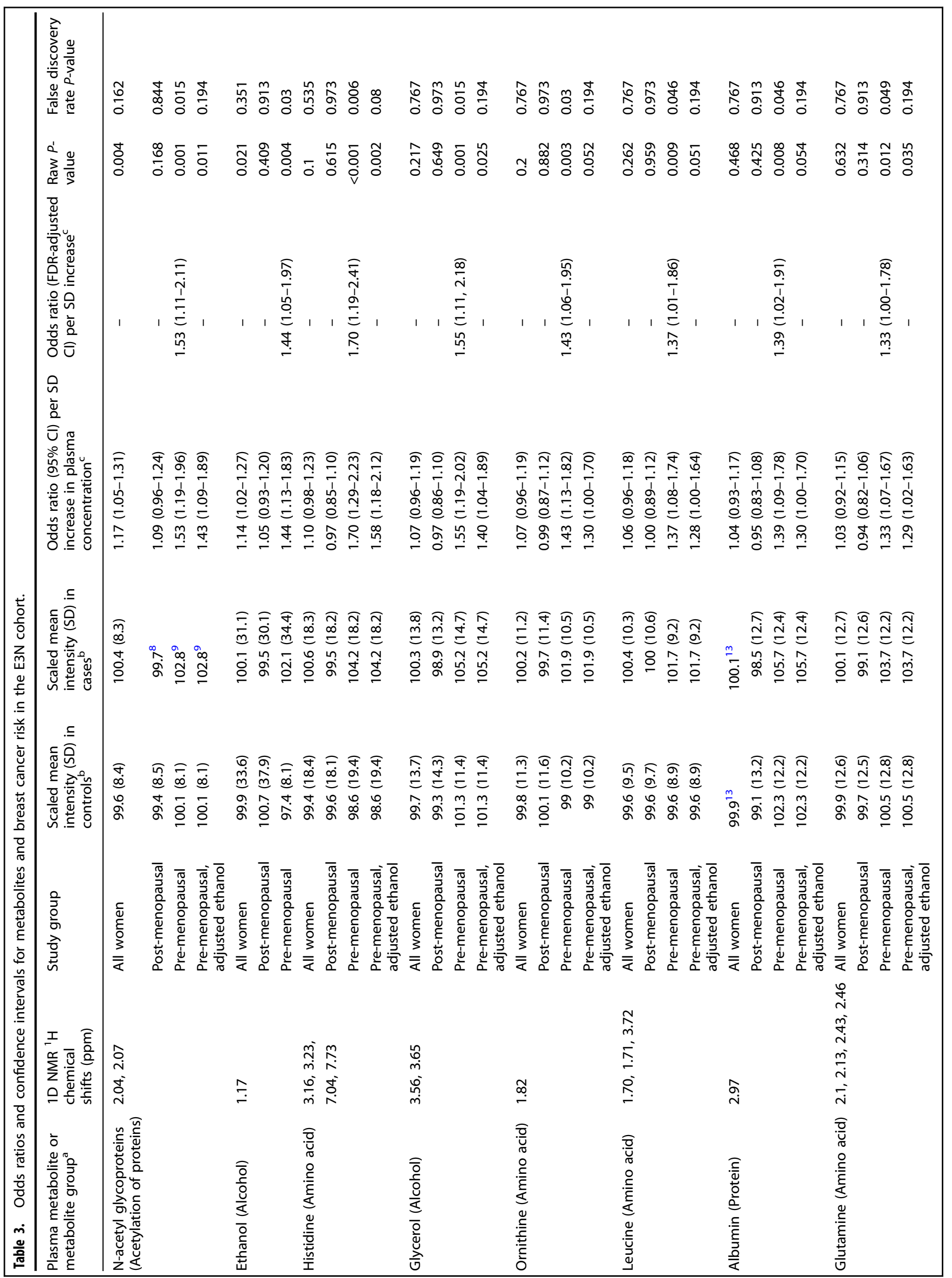


plasma concentrations and breast cancer risk for most other metabolites disappeared or became weaker when plasma ethanol was included as an additional covariate in these models. Therefore, the possibility of residual confounding by alcohol intake should be considered.

The strengths of our study were its substantial size and capacity for subgroup analysis, and particularly the inclusion of more than 300 premenopausal breast cancer cases. Originating from a single country, detailed medical and lifestyle data were acquired and processed consistently. All samples were analysed on the same laboratory platform that has proven to be stable and robust, avoiding the need to account for inter-laboratory variability, and sample processing parameters were of negligible influence on metabolomics data. The study is also subject to certain limitations. Firstly, most participants had not fasted prior to blood collection and time since the last meal was not recorded. However, sensitivity analyses suggested little effect of fasting status upon the polar metabolites most strongly associated with premenopausal breast cancer, and the distinction between pre- and postmenopausal associations remained. Blood samples were only available at a single timepoint meaning that the reproducibility of metabolite measurements could not be assessed, although it is likely that most of these endogenous metabolites are biologically reproducible within a 2-year period. ${ }^{40}$ Also, NMR-based metabolomics does not allow the identification of metabolites to the same degree as mass spectrometry-based platforms, and fewer metabolites were included than in other recent studies, representing a small proportion of the metabolome only. The metabolite set was nonetheless diverse and included representatives of important pathways. Finally, associations for some metabolites weakened upon exclusion of diagnoses made within 2 years of baseline, suggesting the presence, to some extent, of reverse causation.

In summary, untargeted plasma NMR profiles at blood collection were modestly predictive of breast cancer in premenopausal women only. However, differential metabolite associations with breast cancer were found for pre- and postmenopausal women. The metabolites most associated with the disease in premenopausal women were correlated to a substantial extent with plasma ethanol, suggesting residual confounding by alcohol intake. Stratification by menopausal status and a more meticulous consideration of alcohol intake, either by measurement error correction or the use of biomarkers, is therefore needed in future studies of the disease.

\section{DISCLAIMER}

Where authors are identified as personnel of the International Agency for Research on Cancer/World Health Organization, the authors alone are responsible for the views expressed in this article and they do not necessarily represent the decisions, policy or views of the International Agency for Research on Cancer/World Health Organization.

\section{ACKNOWLEDGEMENTS}

We acknowledge Françoise Clavel for her role in the conception and initiation of the study, and Anne Fages for advice on statistical methods. We thank all women participating in the E3N cohort study run by The French National Institute for Medical Research (Inserm) and acknowledge the continuing support of the MGEN, the Institut Gustave Roussy and the French League Against Cancer (Ligue Contre le Cancer).

\section{AUTHOR CONTRIBUTIONS}

L.D., L.B., O.T., M.C.B.R. and B.E. designed the research. E.J. performed laboratory analyses. E.J. and J.R. analysed the data and drafted the paper. B.E. oversaw laboratory analyses. M.F. provided statistical support. L.D., L.L., F.R.M., M.G., G.S. and B. E. gave input into the study design and analysis and interpretation of results. B.E., G.S. and J.R. took primary responsibility for the contents of the paper. 


\section{ADDITIONAL INFORMATION}

Ethics approval and consent to participate The E3N cohort was granted ethical approval by the French National Commission for Computed Data and Individual Freedom (Commission Nationale de I'Informatique et des Libertés) and all E3N participants provided written consent for the use of their blood samples and all data. The study was performed in accordance with the Declaration of Helsinki.

\section{Consent to publish Not applicable.}

Data availability Data are available from the corresponding author upon reasonable request.

Competing interests The authors declare no competing interests.

Funding information This study was funded through the LYriCAN grant from INCaDGOS-Inserm (Grant No. 12563).

Supplementary information The online version contains supplementary material available at https://doi.org/10.1038/s41416-021-01304-1.

Publisher's note Springer Nature remains neutral with regard to jurisdictional claims in published maps and institutional affiliations.

\section{REFERENCES}

1. Bray, F., Ferlay, J., Soerjomataram, I., Siegel, R. L., Torre, L. A. \& Jemal, A. Global cancer statistics 2018: GLOBOCAN estimates of incidence and mortality worldwide for 36 cancers in 185 countries. CA Cancer J. Clin. 68, 394-424 (2018).

2. Continuous Update Project Report: Diet, Nutrition, Physical Activity and Breast Cancer. Available at: wcrf.org/breast-cancer-2017. All CUP reports are available at wcrf.org/cupreports (2017).

3. Cottet, V., Touvier, M., Fournier, A., Touillaud, M. S., Lafay, L., Clavel-Chapelon, F. et al. Postmenopausal breast cancer risk and dietary patterns in the E3N-EPIC prospective cohort study. Am. J. Epidemiol. 170, 1257-1267 (2009).

4. Kesse-Guyot, E., Bertrais, S., Duperray, B., Arnault, N., Bar-Hen, A., Galan, P. et al. Dairy products, calcium and the risk of breast cancer: results of the French SU.VI. MAX prospective study. Ann. Nutr. Metab. 51, 139-145 (2007).

5. Putti, T. C., Abd El-Rehim, D. M., Rakha, E. A., Paish, C. E., Lee, A. H. S., Pinder, S. E. et al. Estrogen receptor-negative breast carcinomas: a review of morphology and immunophenotypical analysis. Mod. Pathol. 18, 26-35 (2005).

6. Tehard, B., Lahmann, P. H., Riboli, E. \& Clavel-Chapelon, F. Anthropometry, breast cancer and menopausal status: Use of repeated measurements over 10 years of follow-up - Results of the french E3N women's cohort study. Int J. Cancer 111, 264-269 (2004).

7. Bro, R., Kamstrup-Nielsen, M. H., Engelsen, S. B., Savorani, F., Rasmussen, M. A., Hansen, L. et al. Forecasting individual breast cancer risk using plasma metabolomics and biocontours. Metabolomics 11, 1376-1380 (2015).

8. Kuhn, T., Floegel, A., Sookthai, D., Johnson, T., Rolle-Kampczyk, U., Otto, W. et al. Higher plasma levels of lysophosphatidylcholine $18: 0$ are related to a lower risk of common cancers in a prospective metabolomics study. BMC Med. 14, 13 (2018).

9. His, M., Viallon, V., Dossus, L., Gicquiau, A., Achaintre, D., Scalbert, A., et al. Prospective analysis of circulating metabolites and breast cancer in EPIC. BMC Med. 17, 178 (2019).

10. Playdon, M. C., Ziegler, R. G., Sampson, J. N., Stolzenberg-Solomon, R., Thompson, H. J., Irwin, M. L. et al. Nutritional metabolomics and breast cancer risk in a prospective study. Am. J. Clin. Nutr. 106, 637-649 (2017).

11. Lecuyer, L., Bala, A. V., Deschasaux, M., Bouchemal, N., Triba, M. N., Vasson, M. P. et al. NMR metabolomic signatures reveal predictive plasma metabolites associated with long-term risk of developing breast cancer. Int J. Epidemiol. 47, 484-494 (2018).

12. Moore, S. C., Playdon, M. C., Sampson, J. N., Hoover, R. N., Trabert, B., Matthews, C. E. et al. A metabolomics analysis of body mass index and postmenopausal breast cancer risk. J. Natl Cancer Inst. 110, 588-597 (2018).

13. Lecuyer, L., Dalle, C., Lyan, B., Demidem, A., Rossary, A., Vasson, M. P. et al. Plasma metabolomic signatures associated with long-term breast cancer risk in the SU.VI.MAX prospective cohort. Cancer Epidemiol. Biomark. Prev. 28, 1300-1307 (2019).

14. Clavel-Chapelon, F., Jadand, C., Goulard, H. \& Guibout-Peigne, C. E3N, a cohort study on cancer risk factors in MGEN women. Description of protocol, main characteristics and population. Bull. Cancer 83, 1008-1013 (1996).
15. Clavel-Chapelon, F. Cohort profile: the French E3N cohort study. Int J. Epidemiol. 44, 801-809 (2015).

16. Guibout-Peigne, C., Prisse, N. \& Clavel-Chapelon, F. A biological bank in an epidemiologic study in France: How? Example of the E3N-EPIC study. Ann. Biol. Clin. 60, 293-298 (2002).

17. Fages, A., Duarte-Salles, T., Stepien, M., Ferrari, P., Fedirko, V., Pontoizeau, C., et al. Metabolomic profiles of hepatocellular carcinoma in a European prospective cohort. BMC Med 13, 242 (2015).

18. Perrier, F., Novoloaca, A., Ambatipudi, S., Baglietto, L., Ghantous, A., Perduca, V., et al. Identifying and correcting epigenetics measurements for systematic sources of variation. Clin. Epigenetics 10, 38 (2018).

19. Kuhn, M. Building predictive models in R using the caret package. J. Stat. Softw. 28, 1-26 (2008)

20. Benjamini, Y. \& Yekutieli, D. False discovery rate-adjusted multiple confidence intervals for selected parameters. J. Am. Stat. Assoc. 100, 71-81 (2005).

21. Touillaud, M. S., Thiebaut, A. C. M., Fournier, A., Niravong, M., Boutron-Ruault, M. C. \& Clavel-Chapelon, F. Dietary lignan intake and postmenopausal breast cancer risk by estrogen and progesterone receptor status. J. Natl Cancer Inst. 99, 475-486 (2007).

22. Jenkins, B. J., Seyssel, K., Chiu, S., Pan, P. H., Lin, S. Y., Stanley, E., et al. Odd chain fatty acids; new insights of the relationship between the gut microbiota, dietary intake, biosynthesis and glucose intolerance. Sci Rep 7, 44845 (2017).

23. Forouhi, N. G., Koulman, A., Sharp, S. J., Imamura, F., Kroger, J., Schulze, M. B. et al. Differences in the prospective association between individual plasma phospholipid saturated fatty acids and incident type 2 diabetes: the EPIC-InterAct casecohort study. Lancet Diabetes Endocrinol. 2, 810-818 (2014).

24. Fuertes-Martin, R., Correig, X., Vallve, J. C. \& Amigo, N. Human serum/plasma glycoprotein analysis by $\mathrm{H}-1-\mathrm{NMR}$, an emerging method of inflammatory assessment. J. Clin. Med. 9, 354 (2020).

25. Lawler, P. R., Akinkuolie, A. O., Chandler, P. D., Moorthy, M. V., Vandenburgh, M. J., Schaumberg, D. A. et al. Circulating N-linked glycoprotein acetyls and longitudinal mortality risk. Circ. Res. 118, 1106-1115 (2016).

26. Medina, V., Cricco, G., Nunez, M., Martin, G., Mohamad, N., Correa-Fiz, F. et al. Histamine-mediated signaling processes in human malignant mammary cells. Cancer Biol. Ther. 11, 1462-1471 (2006).

27. Greenfield, J. R., Farooqi, I. S., Keogh, J. M., Henning, E., Habib, A. M., Blackwood, A. et al. Oral glutamine increases circulating glucagon-like peptide 1, glucagon, and insulin concentrations in lean, obese, and type 2 diabetic subjects. Am. J. Clin. Nutr. 89, 106-113 (2009).

28. Bai, D. \& Song, J. N. Study of metabolic biomarkers in plasma of patients with dyslipidemia based on gas chromatography-mass spectrometry and graphical models. Chin. J. Anal. Chem. 40, 1482-1487 (2012).

29. Wang, Q., Ferreira, D. L. S., Nelson, S. M., Sattar, N., Ala-Korpela M. \& Lawlor D. A. Metabolic characterization of menopause: cross-sectional and longitudinal evidence. BMC Med. 16, 17 (2018).

30. Sun, Q., Xie, W., Wang, Y., Chong, F., Song, M. \& Li T. et al. Alcohol consumption by beverage type and risk of breast cancer: a dose-response Meta-analysis of prospective cohort studies. Alcohol Alcohol 55, 246-253.(2020).

31. Fagherazzi, G., Vilier, A., Boutron-Ruault, M. C., Mesrine, S. \& Clavel-Chapelon, F. Alcohol consumption and breast cancer risk subtypes in the E3N-EPIC cohort. Eur. J. Cancer Prev. 24, 209-214 (2015).

32. Seitz, H. K. \& Stickel, F. Molecular mechanisms of alcohol-mediated carcinogenesis. Nat. Rev. Cancer 7, 599-612 (2007).

33. van Roekel, E. H., Trijsburg, L., Assi, N., Carayol, M., Achaintre, D., Murphy, N. et al. Circulating metabolites associated with alcohol intake in the European Prospective Investigation into Cancer and Nutrition Cohort. Nutrients 10, 654 (2018).

34. Lacruz, M. E., Kluttig, A., Tiller, D., Medenwald, D., Giegling, I., Rujescu, D. et al. Cardiovascular risk factors associated with blood metabolite concentrations and their alterations during a 4-year period in a population-based cohort. Circ. Cardiovasc. Genet. 9, 487-494 (2016).

35. Jaremek, M., Yu, Z., Mangino, M., Mittelstrass, K., Prehn, C., Singmann, P. et al. Alcoholinduced metabolomic differences in humans. Transl. Psychiatry 3, e276 (2013).

36. Harada, S., Takebayashi, T., Kurihara, A., Akiyama, M., Suzuki, A., Hatakeyama, Y. et al. Metabolomic profiling reveals novel biomarkers of alcohol intake and alcohol-induced liver injury in community-dwelling men. Environ. Health Prev. Med. 21, 18-26 (2016).

37. Pallister T., Jennings A., Mohney R. P., Yarand D., Mangino M., Cassidy A. et al. Characterizing blood metabolomics profiles associated with self-reported food intakes in female twins. PLOS ONE 11, e0158568 (2016).

38. Irwin, C., Van Reenen, M., Mason, S., Mienie, L. J., Wevers, R. A., Westerhuis, J. A., et al. The H-1-NMR-based metabolite profile of acute alcohol consumption: A metabolomics intervention study. PLOS ONE 13, e0196850 (2018). 
Investigation of circulating metabolites associated with breast cancer... E Jobard et al.

39. Klatsky, A. L., Udaltsova, N., Li, Y., Baer, D., Tran, H. N. \& Friedman, G. D. Moderate alcohol intake and cancer: the role of underreporting. Cancer Causes Control 25, 693-699 (2014).

40. Carayol, M., Licaj, I., Achaintre, D., Sacerdote, C., Vineis, P., Key, T. J. et al. Reliability of serum metabolites over a two-year period: a targeted metabolomic approach in fasting and non-fasting samples from EPIC. PLOS ONE 10, e0135437 (2015).

(i) Open Access This article is licensed under a Creative Commons Attribution 4.0 International License, which permits use, sharing, adaptation, distribution and reproduction in any medium or format, as long as you give appropriate credit to the original author(s) and the source, provide a link to the Creative Commons license, and indicate if changes were made. The images or other third party material in this article are included in the article's Creative Commons license, unless indicated otherwise in a credit line to the material. If material is not included in the article's Creative Commons license and your intended use is not permitted by statutory regulation or exceeds the permitted use, you will need to obtain permission directly from the copyright holder. To view a copy of this license, visit http://creativecommons. org/licenses/by/4.0/.

(c) The Author(s) 2021 